\title{
The Toho Score: A New Evaluation System for Chronic Sinusitis Using Computed Tomography with Ziostation
}

\author{
Yasushi Ota ${ }^{1}$, Masayuki Odashima ${ }^{2}$, Hitoshi Terada ${ }^{2}$, Toshiyuki Nomura ${ }^{1}$, Tamura Yuuya ${ }^{1}$, Hiroyoshi Ikemiyagi ${ }^{1}$ \\ and Mitsuya Suzuki ${ }^{1}$ \\ 1. Departments of Otorhinolaryngology, Toho University Sakura Medical Center, Chiba 285-8741, Japan \\ 2. Departments of Radiology, Toho University Sakura Medical Center, Chiba 285-8741, Japan
}

\begin{abstract}
OBJECTIVE: We tried a new system for the evaluation of chronic sinusitis (CS) that uses a Computed tomography (CT) system equipped with the Ziostation 2 (Zio 2). SUBJECTS AND METHODS: Subjects were selected from patients with CS who had been evaluated using a CT system equipped with the Zio 2. Participants included 10 CS patients, 20 sides. The percentage of the volume of the soft-tissue density area relative to the total volume (STDA\%) was calculated using Zio 2 system. We named this STDA\% determined using the Zio 2 system the "Toho score.” Two doctors carried out these evaluations, and their results were compared. Toho score was compared with the Lund-Mackay (L-M) score. RESULTS: The Toho scores were correlated between the two doctors with significance (the correlation coefficients of total paranasal sinus area, 0.95). They were correlated with the corresponding L-M score values with significance, too (the correlation coefficients of total paranasal sinus area, 0.82). CONCLUSION: Toho system may be useful for accurately determining the extent of the inflammatory lesion in the paranasal sinus. In future, it can be employed effectively for monitoring treatment effects in chronic sinusitis and other conditions that involve inflammation of the paranasal sinuses.
\end{abstract}

Key words: The Lund-Mackay (L-M) score, computer workstation, paranasal sinus.

\section{Introduction}

Computed tomography (CT) is a useful tool for assessing the volume of inflammatory load within the paranasal sinuses in chronic sinusitis (CS) and objectively evaluating the degree of improvement in CS before and after drug therapy or surgery [1-4]. The Lund-Mackay system [4] (L-M system) is widely used in CT evaluation of CS because of its convenience. However, it is often pointed out that this system lacks sufficient levels of gradation for tracking progression or reduction of the disease volume [5]. Therefore, we intended to measure soft tissue shadow in the CT of the paranasal sinus more exactly.

In this study, we investigated a new system for the evaluation of CS that uses a CT system equipped with the Ziostation 2 (Zio 2). We can easily select the space of the paranasal sinus using Zio 2 and can know that's

Corresponding author: Yasushi Ota, MD, Ph.D., associate professor, research field: nasal and paranasal disease, allergy, sinusitis, IgE, IgG4. volume. Fig. 1 shows the both sides of the whole paranasal sinus of a normal case. This total volume was $55 \mathrm{~mL}$.

\section{Subjects and Methods}

\subsection{Subjects}

Subjects were selected from patients with CS who had been evaluated using a CT system equipped with the Zio 2 at the Toho Sakura Medical Center Otorhinolaryngology Department from July 2014 through March 2015. CS was diagnosed from the clinical symptoms such as nasal obstruction, rhinorrhea, a postnasal drip, smell disorder, headaches, and local site views such as nasal polyps and CT image views according to diagnostic criteria of the Japanese rhinology society. All cases were preoperative cases, and there was not the case after the nose and paranasal operation. 


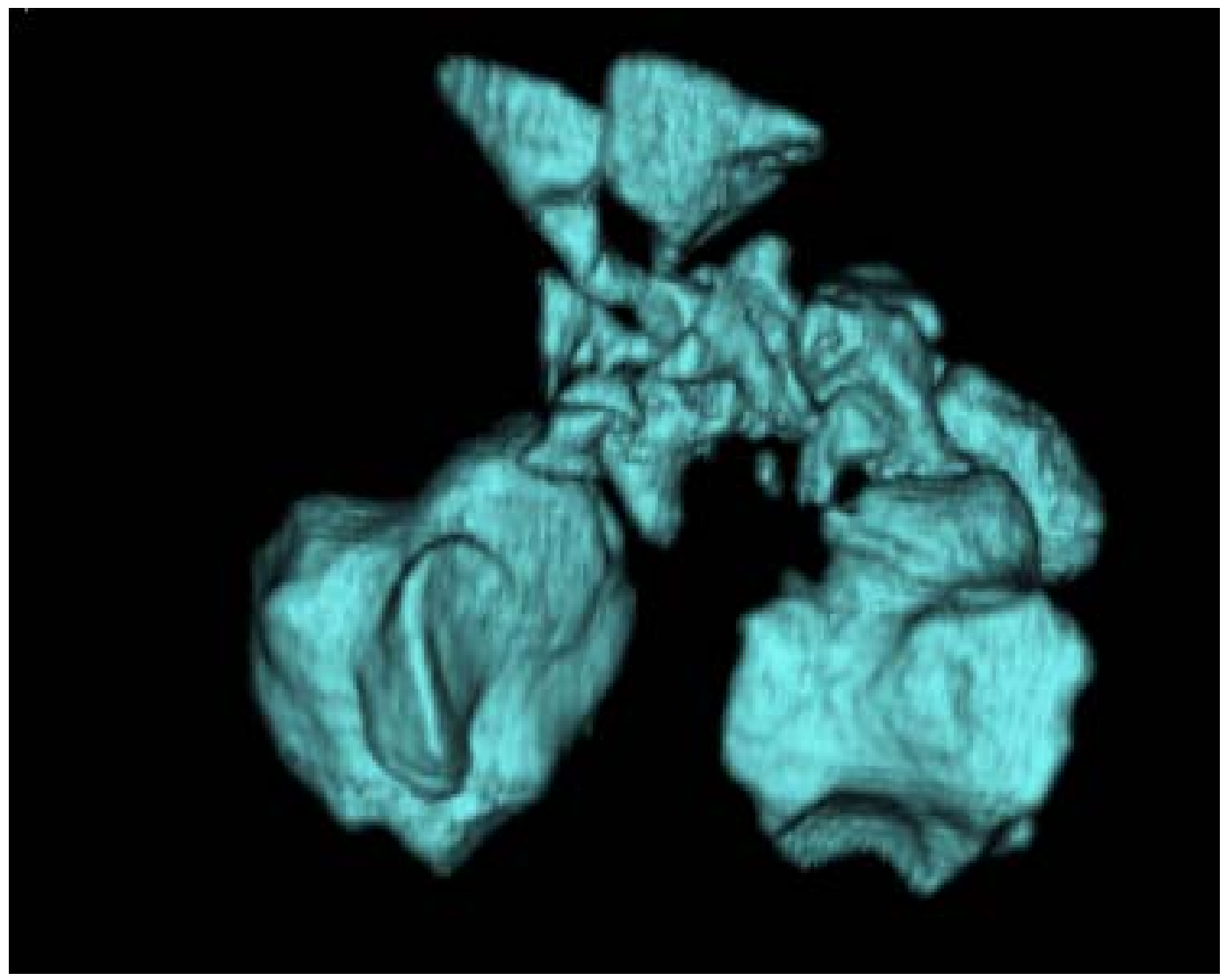

Fig. 1 The both sides of the whole paranasal sinus of one normal case.

\subsection{Methods}

Axial, coronal, and sagittal images were acquired preoperatively with a multi-slice CT helical scanning system (Aquilion 64; Toshiba medical systems corporation, Japan) with the following parameters: 120 $\mathrm{kV}, 200 \mathrm{~mA}$. To quantify the volume of inflammatory opacification, the soft-tissue-density and air volumes in each paranasal sinus were assessed with a computer workstation, the Zio 2 (Ziosoft, Tokyo, Japan).

First, we used the expansion system of the Zio 2 to measure the volume of air in each sinus cavity - the maxillary, sphenoid, frontal, and ethmoid sinuses (Fig. 2). Next, we measured the volume of soft-tissue density in each paranasal sinus, using the same method (Fig. 3). The sum of the volumes of these two parts, the air and soft-tissue density in each sinus, was considered to represent the total volume of the sinus. The percentage of the volume of the soft-tissue density area
(STDA) relative to the total volume (STDA\%) was calculated as follows: volume of the soft-tissue density area/total volume of the sinus $\times 100$. Two doctors carried out these evaluations, and their results were compared. In addition, we measured how much time these measurements required. We named this STDA\% determined using the Zio 2 system the "Toho score."

To evaluate the legitimacy of the score for each sinus area, the Toho score was compared with the score assigned in accordance with the L-M system. We examined correlations and disagreements between the results of the two scoring systems for the total paranasal sinus area and each paranasal sinus (maxillary, sphenoid, frontal, and ethmoid) for 20 sides in 10 cases.

\subsection{Data Analysis}

The correlations between the Toho scores of the two doctors, and between the Toho score and L-M score for 


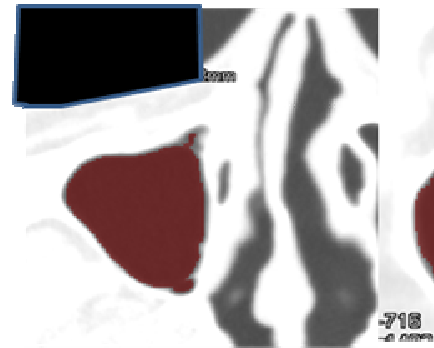

a

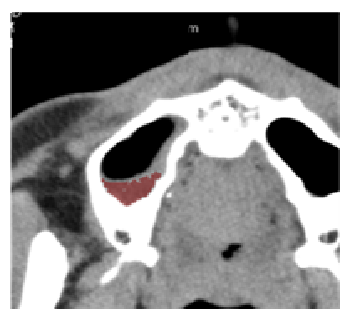

e

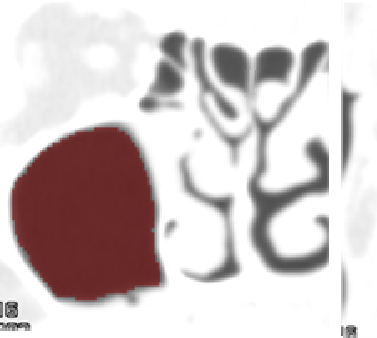

b

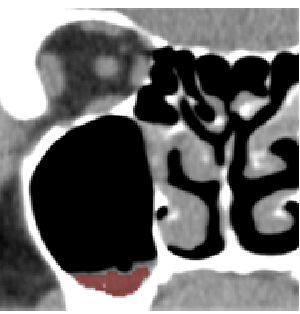

f

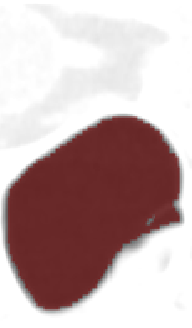

c

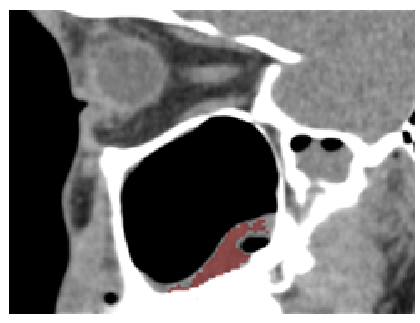

g

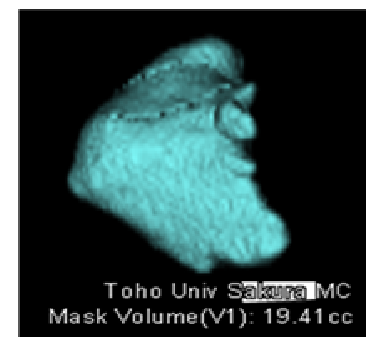

d

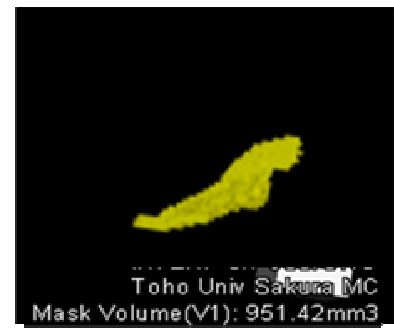

h

Fig. 2 Measurement of the air and soft-tissue-density volumes in a maxillary sinus.

First, the quantity of the air in the right maxillary sinus is determined. A point representative of air is selected in the horizontal, coronal, and sagittal section images (Fig. 2-a, b, c). With the expansion function of Zio 2, the image is automatically extended from the point to the whole area with the same CT level. Finally, a pictorial drawing of the air area of the right maxillary sinus is generated and the volume of the area is measured (Fig. 2-d).

The volume of the low-density area of the same maxillary sinus is determined in the same way (Fig. 1-e, f, g, h).

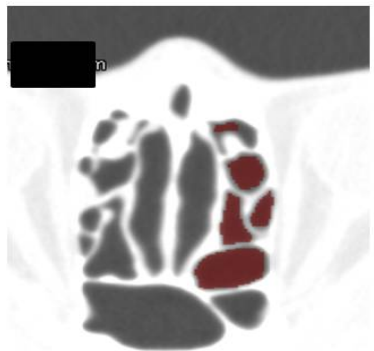

a

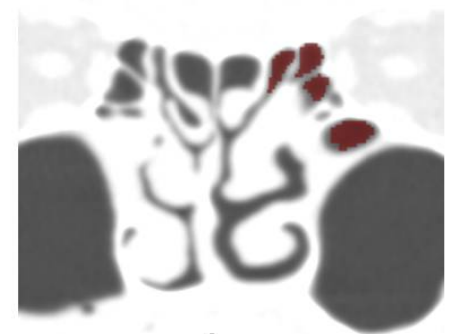

b

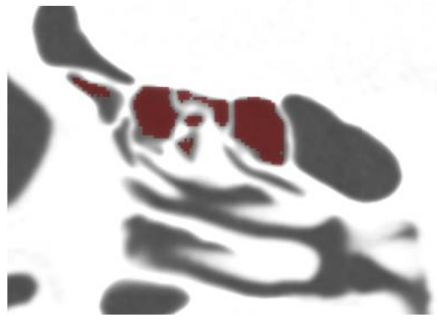

c

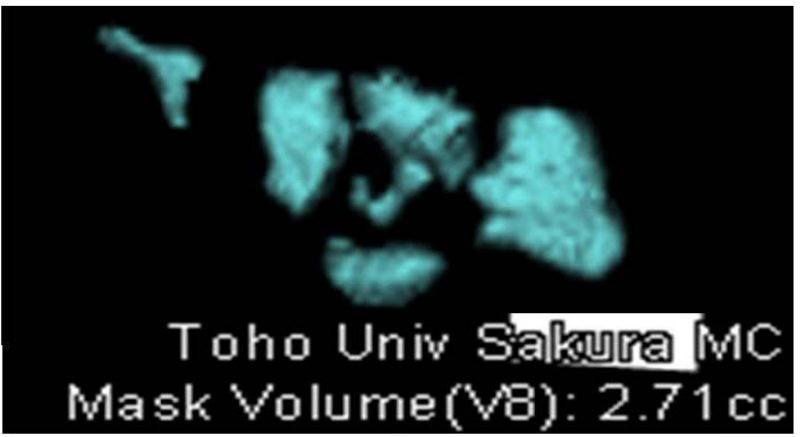

d

Fig. 3 Measurement of the ethmoid sinus with the Toho system.

This sinus comprises multiple small areas (Fig. 3-a, b, c, d). 
the total paranasal sinus area and each paranasal sinus, were evaluated using Pearson's correlation coefficient.

\section{Results}

3.1 Measurement Results of the Toho Score and L-M Score

Participants included 10 CS patients (7 male and 3 female patients) with a median age of 53 years (range, 35-71 years). The Toho score and L-M score results of the two doctors for the total paranasal sinus area and each paranasal sinus in each patient are shown in Table 1.

3.2 Correlation between Toho Score Values of the Two Doctors

The Toho scores for the total paranasal sinus area, maxillary sinus, sphenoid sinus, frontal sinus, and ethmoid sinus were correlated between the two doctors with significance (Fig. 4). The correlation coefficients between the two doctors were as follows: total paranasal sinus area, 0.95; maxillary sinus, 0.99; sphenoid sinus, 0.99; frontal sinus, 0.97; and ethmoid sinus, 0.83 .

3.3 Correlations between the Toho Score and L-M Score Values

The Toho score values for the total paranasal sinus area, maxillary sinus, sphenoid sinus, frontal sinus, and ethmoid sinus were correlated with the corresponding L-M score values with significance (Fig. 5). The correlation coefficients between the two scoring systems were as follows: total paranasal sinuses area, 0.82; maxillary sinus, 0.61; sphenoid sinus, 0.72; frontal sinus, 0.74; and ethmoid sinus, 0.64.

Table 1 Measurement results of the Toho score and L-M score.

\begin{tabular}{|c|c|c|c|c|c|c|c|}
\hline & & Toho score R Dr1 & Toho score R Dr2 & L-M score R & Toho score L Dr1 & Toho score L Dr2 & L-M score L \\
\hline \multirow{6}{*}{ Case 1} & maxillary sinus & 100 & 100 & 2 & 35.5 & 31.32 & 1 \\
\hline & ethmoid sinus & 100 & 100 & 4 & 41.3 & 22.62 & 2 \\
\hline & sphenoid sinus & 100 & 100 & 2 & 0 & 0 & 0 \\
\hline & frontal sinus & 100 & 100 & 2 & 0 & 0 & 0 \\
\hline & OMU & & & 2 & & & 2 \\
\hline & total & 100 & 100 & 12 & 32.1 & 21.28 & 5 \\
\hline \multirow{6}{*}{ Case 2} & maxillary sinus & 17.99 & 22.03 & 1 & 35.49 & 57.17 & 1 \\
\hline & ethmoid sinus & 69.45 & 50.02 & 2 & 56.91 & 61.11 & 2 \\
\hline & sphenoid sinus & 11.7 & 11.56 & 1 & 37.48 & 34.85 & 1 \\
\hline & frontal sinus & 94.84 & 98.73 & 1 & 7.95 & 22 & 1 \\
\hline & OMU & & & 2 & & & 2 \\
\hline & total & 21.83 & 27.07 & 7 & 36.04 & 50.9 & 7 \\
\hline \multirow{6}{*}{ Case 3} & maxillary sinus & 0 & 0 & 0 & 100 & 100 & 2 \\
\hline & ethmoid sinus & 0 & 0 & 0 & 0 & 0 & 1 \\
\hline & sphenoid sinus & 0 & 0 & 0 & 0.16 & 0 & 0 \\
\hline & frontal sinus & 0 & 0 & 0 & 0 & 0 & 0 \\
\hline & OMU & & & 0 & & & 2 \\
\hline & total & 0 & 0 & 0 & 58.72 & 33.04 & 5 \\
\hline \multirow{6}{*}{ Case 4} & maxillary sinus & 0.6 & 0.73 & 0 & 3.65 & 6.31 & 1 \\
\hline & ethmoid sinus & 14.98 & 12.56 & 1 & 22.01 & 13.73 & 1 \\
\hline & sphenoid sinus & 3.75 & 3.94 & 1 & 8.22 & 13.04 & 1 \\
\hline & frontal sinus & 17.34 & 37.46 & 1 & 61.08 & 81.64 & 1 \\
\hline & OMU & & & 0 & & & 0 \\
\hline & total & 4.69 & 5.5 & 3 & 11.46 & 17.15 & 4 \\
\hline
\end{tabular}


The Toho Score: A New Evaluation System for Chronic Sinusitis Using Computed Tomography with Ziostation

(Table 1 continued)

\begin{tabular}{|c|c|c|c|c|c|c|c|}
\hline & & Toho score R Dr1 & Toho score R Dr2 & L-M score $\mathrm{R}$ & Toho score L Dr1 & Toho score L Dr2 & L-M score L \\
\hline \multirow{6}{*}{ Case 5} & $\begin{array}{l}\text { maxillary } \\
\text { sinus }\end{array}$ & 1.81 & 1.4 & 1 & 96.78 & 98.79 & 1 \\
\hline & ethmoid sinus & 67.6 & 58.7 & 2 & 13.07 & 50.83 & 2 \\
\hline & sphenoid sinus & 1.04 & 0.79 & 1 & 62.69 & 77.29 & 1 \\
\hline & frontal sinus & 100 & 100 & 1 & 93.16 & 88.84 & 1 \\
\hline & OMU & & & 1 & & & 1 \\
\hline & total & 20.04 & 14.36 & 6 & 80.55 & 87.39 & 6 \\
\hline \multirow{6}{*}{ Case 6} & $\begin{array}{l}\text { maxillary } \\
\text { sinus }\end{array}$ & 87.85 & 82.92 & 1 & 89.58 & 88.34 & 1 \\
\hline & ethmoid sinus & 65.43 & 81.58 & 2 & 84.18 & 83.59 & 2 \\
\hline & sphenoid sinus & 0 & 0.73 & 1 & 61.73 & 75.8 & 1 \\
\hline & frontal sinus & 98.42 & 99.13 & 2 & 100 & 100 & 2 \\
\hline & OMU & & & 2 & & & 2 \\
\hline & total & 67.58 & 64.65 & 8 & 86.92 & 89.88 & 8 \\
\hline \multirow{6}{*}{ Case 7} & $\begin{array}{l}\text { maxillary } \\
\text { sinus }\end{array}$ & 49.56 & 61.5 & 1 & 87.55 & 97.56 & 2 \\
\hline & ethmoid sinus & 0 & 1 & 2 & 0 & 18.56 & 2 \\
\hline & sphenoid sinus & 0 & 0 & 0 & 0 & 0 & 0 \\
\hline & frontal sinus & 0 & 0 & 0 & 0 & 0 & 0 \\
\hline & OMU & & & 0 & & & 2 \\
\hline & total & 13.7 & 14.24 & 3 & 32.19 & 35.78 & 6 \\
\hline \multirow{6}{*}{ Case 8} & $\begin{array}{l}\text { maxillary } \\
\text { sinus }\end{array}$ & 89.88 & 92.37 & 1 & 4.76 & 4.11 & 1 \\
\hline & ethmoid sinus & 0 & 5.51 & 2 & 0 & 0 & 0 \\
\hline & sphenoid sinus & 0 & 0 & 0 & 0 & 0 & 0 \\
\hline & frontal sinus & 0 & 0 & 1 & 0 & 0 & 0 \\
\hline & OMU & & & 0 & & & 0 \\
\hline & total & 39.48 & 42.18 & 4 & 3.03 & 2.63 & 1 \\
\hline \multirow{6}{*}{ Case 9} & $\begin{array}{l}\text { maxillary } \\
\text { sinus }\end{array}$ & 10.27 & 7.08 & 1 & 0.71 & 0 & 1 \\
\hline & ethmoid sinus & 30.38 & 8.17 & 2 & 23.27 & 76.55 & 2 \\
\hline & sphenoid sinus & 11.21 & 4.89 & 0 & 0 & 0 & 0 \\
\hline & frontal sinus & 32.42 & 2.76 & 1 & 0 & 0 & 0 \\
\hline & OMU & & & 0 & & & 0 \\
\hline & total & 17.1 & 6.25 & 4 & 6.39 & 2.24 & 3 \\
\hline \multirow{6}{*}{ Case 1} & $\begin{array}{l}\text { maxillary } \\
\text { sinus }\end{array}$ & 0.43 & 0.35 & 1 & 9.32 & 3.31 & 1 \\
\hline & ethmoid sinus & 21.33 & 18.42 & 2 & 39.29 & 20.19 & 2 \\
\hline & sphenoid sinus & 0 & 0 & 0 & 0 & 0 & 0 \\
\hline & frontal sinus & 3.03 & 0 & 1 & 0 & 0 & 1 \\
\hline & OMU & & & 0 & & & 0 \\
\hline & total & 3.77 & 1.65 & 4 & 13.34 & 5.01 & 4 \\
\hline
\end{tabular}

OMU: osteomeatal unit.

Dr: doctor.

L: Left side.

R: Right side. 


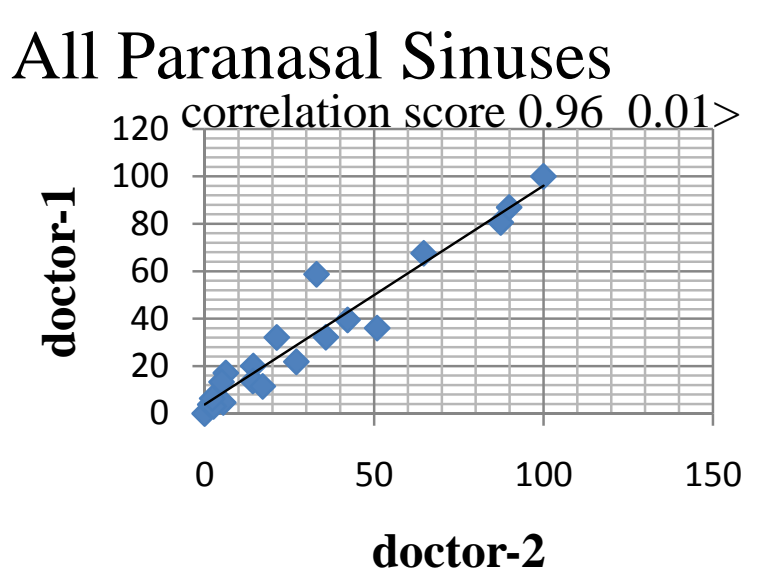

Sphenoid Sinus

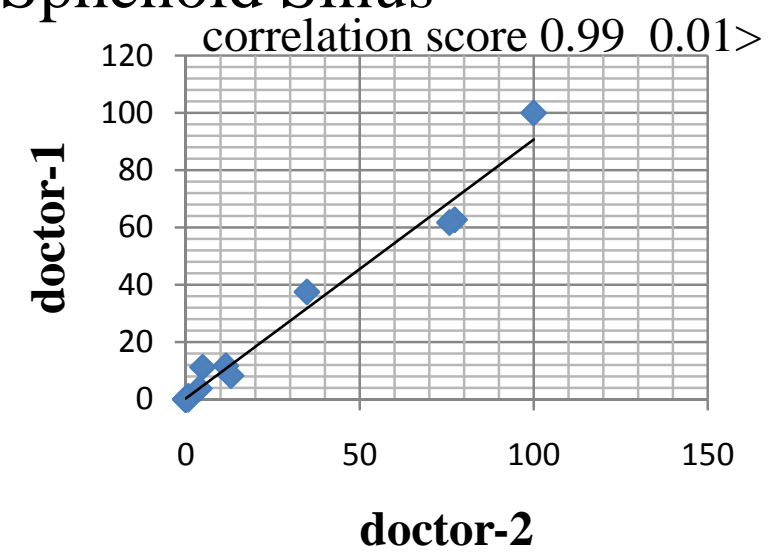

Maxillary Sinus

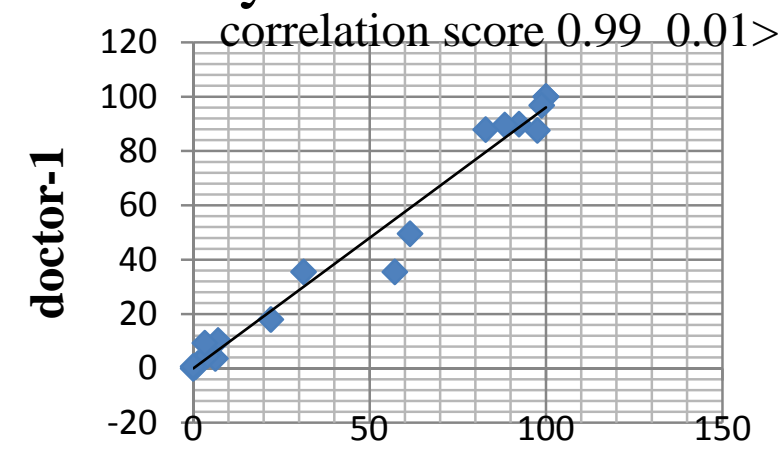

doctor-2

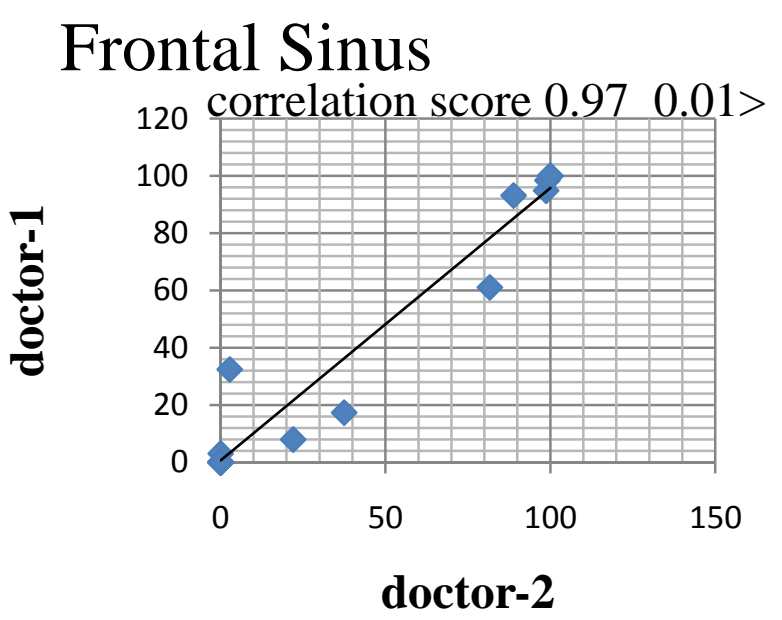

\section{Ethmoid Sinus}

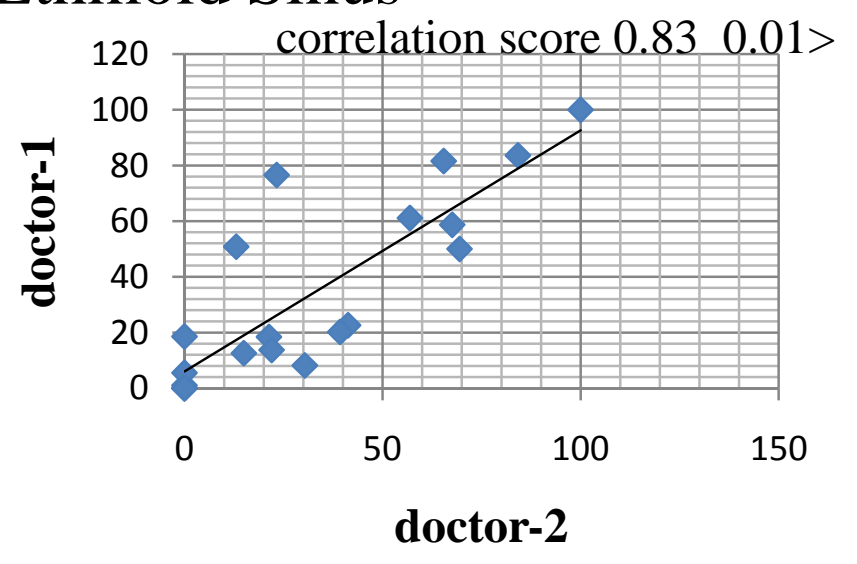

Fig. 4 Correlation between the Toho score results of the two doctors.

The Toho scores of the total paranasal sinus area, maxillary sinus, sphenoid sinus, frontal sinus, and ethmoid sinus were correlated between the two doctors. The correlation coefficients were as follows: total paranasal sinus area, 0.95; maxillary sinus, 0.99; sphenoid sinus, 0.99; frontal sinus, 0.97; and ethmoid sinus, 0.83 . 


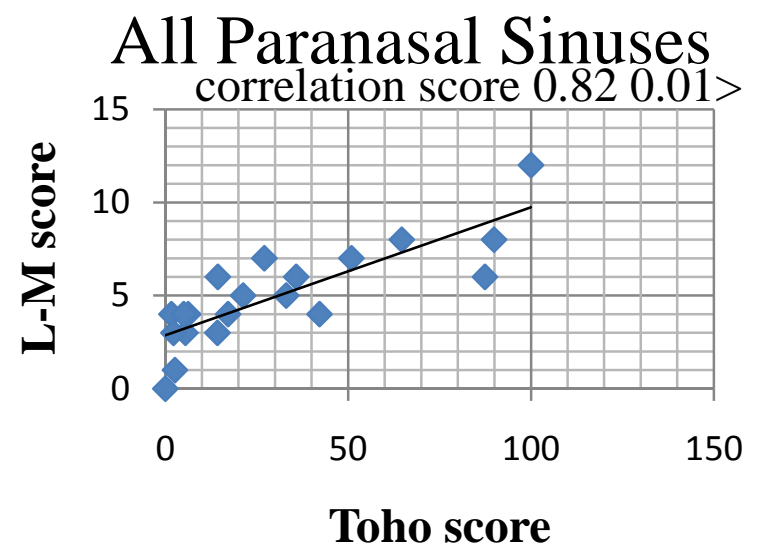

Sphenoid Sinus

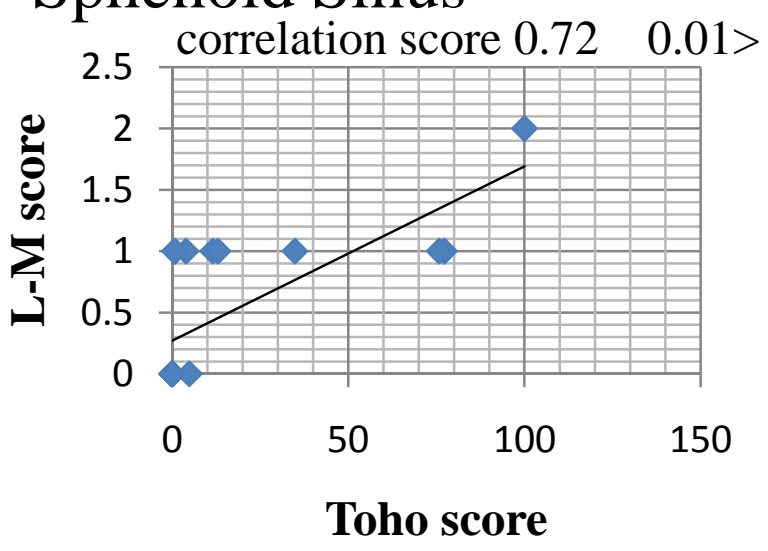

Maxillary Sinus

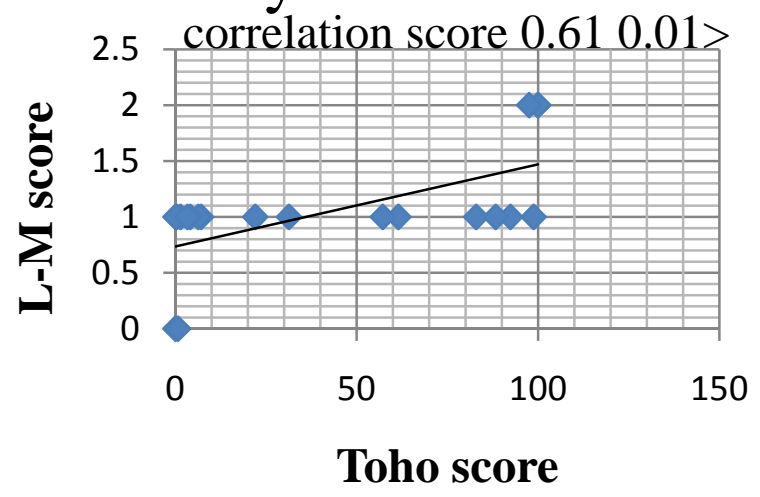

Frontal Sinus

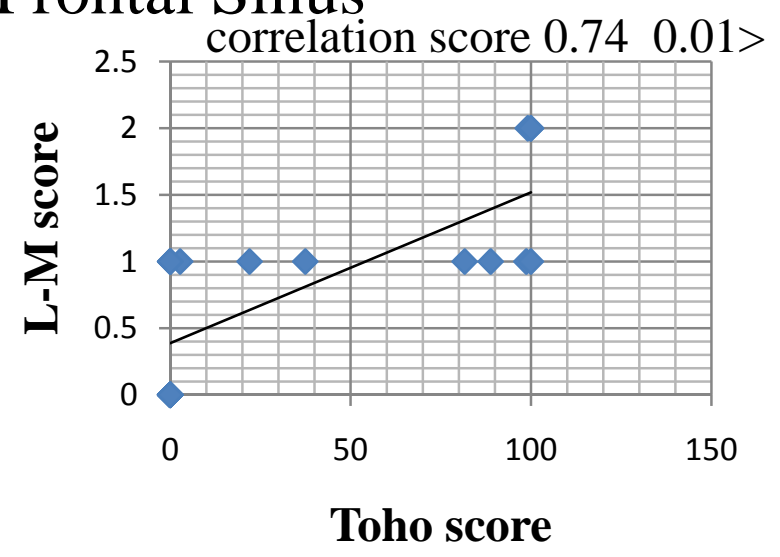

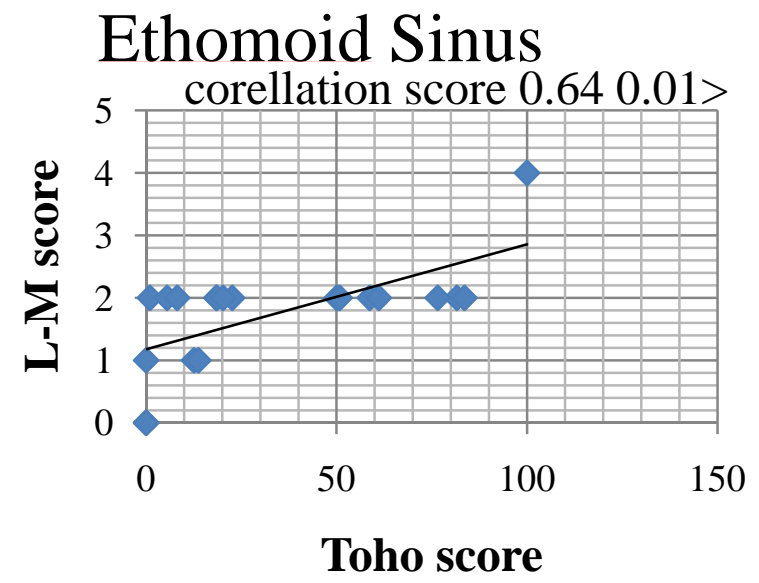

Fig. 5 Correlation between the Toho score and L-M score results.

The Toho scores of the total paranasal sinus area, maxillary sinus, sphenoid sinus, frontal sinus, and ethmoid sinus were correlated with the corresponding L-M scores. The correlation coefficients were as follows: total paranasal sinus, 0.82; maxillary sinus, 0.61; sphenoid sinus, 0.72; frontal sinus, 0.74; and ethmoid sinus, 0.65 . 


\subsection{Work Time}

On average, the two doctors required 35 and 37 minutes for measurements with the Zio 2 and calculation of the Toho scores for each case. The mean time required for determination of the L-M scores was 5 minutes.

\section{Discussion}

In this study, we investigated a new evaluation system using a CT system equipped with the Ziostation 2 (Zio 2) for analyzing CS in 10 patients. We were able to determine the proportion of the paranasal sinus occupied by inflammatory tissue as a percentage (which was named the "Toho score") in all paranasal sinuses of 20 sides of 10 cases. We then compared the results obtained with this system with those obtained with the L-M system.

The L-M system is widely used in CT evaluation of CS. The wide acceptance of the L-M system is due to its simple staging (Table 1) [4]. When sinus inflammation occupies $0 \%$ of the CT image, a score of 0 is assigned, while a score of 2 is assigned when the inflammation occupies $100 \%$ of the image. All other degrees of inflammation are scored as 1 . However, it is often pointed out that this system lacks sufficient levels of gradation for tracking progression or reduction of the disease volume [5]. Three more detailed staging systems, which are modifications of the L-M system, have been reported in recent years to help resolve these deficiencies [5-7].

On the other hand, advances have been made in CT workstation techniques and additional functions have been developed. CT workstations are now used in various medical fields, including cardiac care, as an aid in diagnosis and treatment $[8,9]$. In the paranasal sinus domain, a CT workstation has been used in a study of the approach to the frontal sinus [10]. In the present study, we investigated a new scoring system for inflammatory lesions in the paranasal sinus domain by using the CT workstation "Zio 2."
Our aim was to use the Zio 2 to establish an easy method for evaluation of inflammatory lesions in the paranasal sinuses. The overall volume of each paranasal sinus and the volume of the inflammatory tissue were used to calculate the percentage of the sinus volume occupied by inflammatory tissue. We also measured how much time was required for determination of the scores in each case to gauge the difficulty of using this system.

In this study, we mainly used the expansion function of the Zio 2 for measurements. When a certain point on the paranasal sinus CT image is clicked, the system enlarges the domain having the same CT level and measures the volume of it. This technique is very easy and does not take much time, although accurate selection of the domain requires an appropriate skill level and a small amount of time. It is easier to apply this technique to the large paranasal sinuses, that is, the maxillary, sphenoid, and frontal sinuses, than to the ethmoid sinus with its conglomeration of small cavities.

We were able to determine the percentage of paranasal sinus area occupied by inflammatory tissue (named the "Toho score") in all paranasal sinuses of 20 sides from 10 cases with the Zio 2. In addition, two doctors separately determined the score values. The results of the two doctors were almost completely in accordance. This shows that the method of evaluating paranasal sinus lesions with the Zio 2 is legitimate.

The Toho score results correlated with the corresponding results of the L-M system. This suggests that the Toho score system is legitimate. However, the correlation coefficients between the scores of the two systems for each paranasal sinus (maxillary sinus, 0.61; sphenoidal sinus, 0.72; frontal sinus, 0.65; and ethmoid sinus, 0.65) were lower than that for the whole paranasal sinus (0.82). This result suggests that accuracy declines with the L-M system when we evaluate each individual paranasal sinus.

The L-M system is widely used in CT evaluation of CS because of its convenience [4]. The mean time 
needed for determination of the L-M scores was 5 minutes per case for 20 sides in 10 cases. On the other hand, the two doctors took 35 minutes and 37 minutes to determine the Toho scores in each case. New methods of evaluation are not adopted unless the technique is easier to apply compared with preexisting methods. It is necessary to reduce the time needed to determine the Toho scores to promote this system, and further research focused on devising ways of reducing the operation time is warranted.

In this study, we often found that a wide range of Toho scores corresponded with the same L-M score. For example, as was the case with the patient images shown in Fig. 6, the Toho score of the right maxillary sinus was $89.88 \%$ and of the left was $4.76 \%$, although the L-M score of both sides was 1 . Another case is shown in Fig. 7 in which the Toho score of the right frontal sinus was $98.73 \%$ and of the left was $22 \%$, although the L-M score of both sides was 1 . This illustrates how detailed information may not be available with use of the L-M system because the degree of the lesion can vary widely for an L-M score of 1 . On the other hand, the area of the inflammatory lesion in the paranasal sinus can be represented more accurately with the Toho score, because the Toho score shows the proportion of the paranasal sinus occupied by inflammatory tissue as a percentage. Hence, we believe that the Toho system may be useful for accurately determining treatment effects in paranasal sinus lesions.

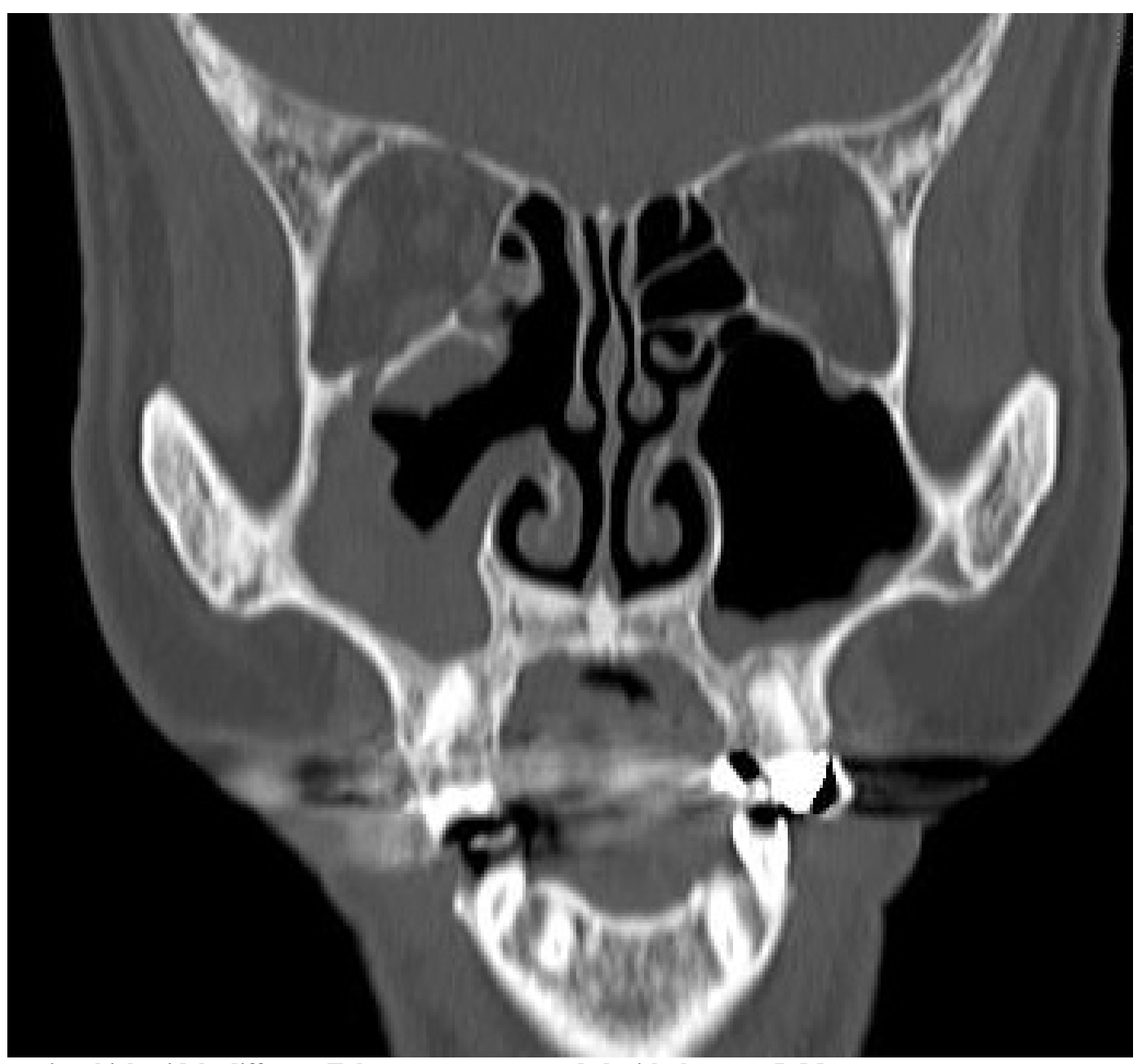

Fig. 6 A case in which widely different Toho scores corresponded with the same L-M score.

The Toho score of the right maxillary sinus was $89.88 \%$ and of the left was $4.76 \%$, although the L-M score of both sides was 1 . 


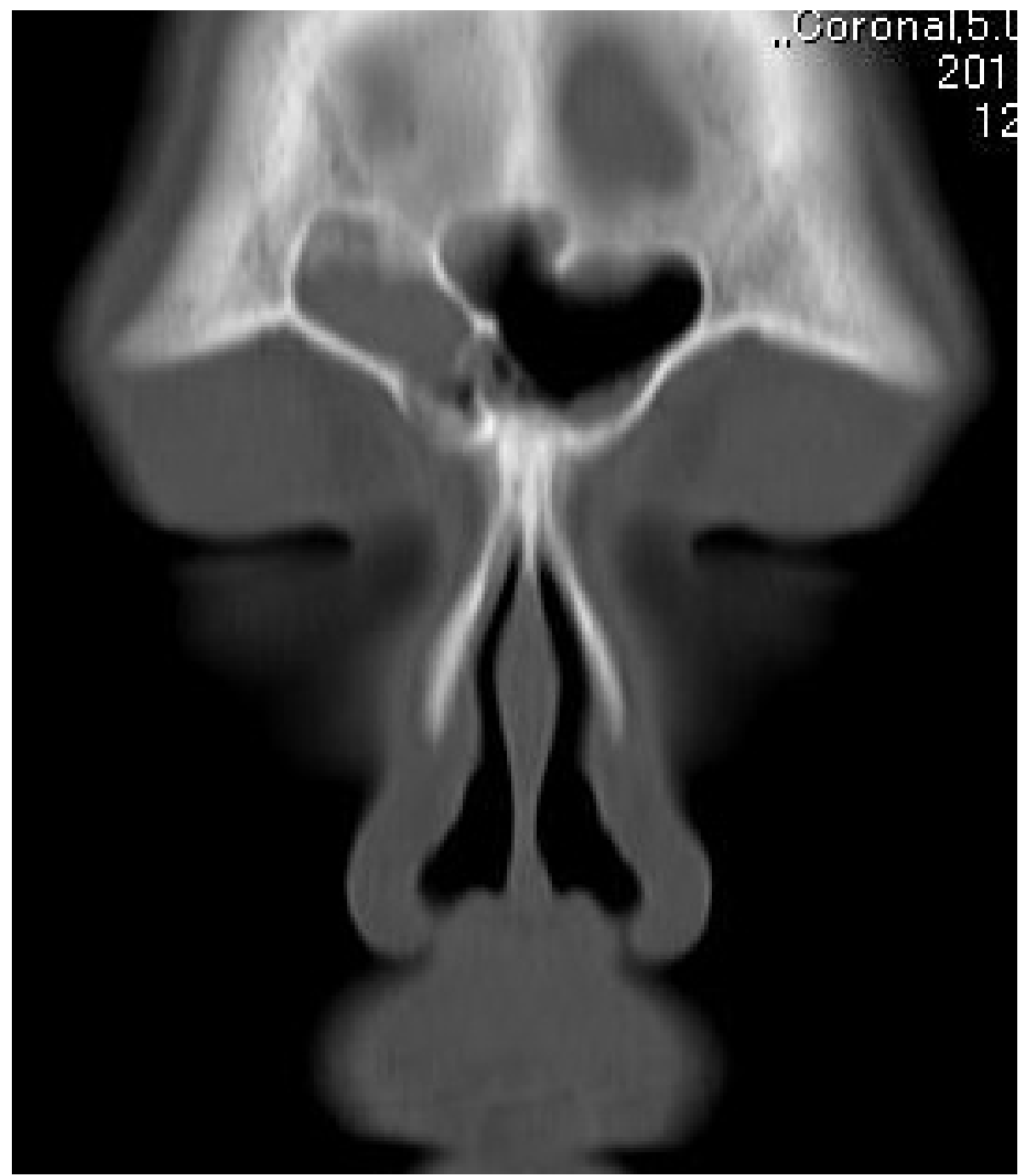

Fig. 7 A case in which widely different Toho scores corresponded with the same L-M score.

The Toho score of the right frontal sinus was $98.73 \%$ and of the left was $22 \%$, although the L-M score of both sides was 1 .

\section{Conclusion}

Toho score values for evaluation of paranasal sinus inflammation show good correlation with the corresponding L-M score values. In addition, Toho scores provided a more detailed and accurate representation of the extent of the inflammatory lesion in the paranasal sinuses, as compared to L-M scores. Thus, we conclude that the Toho system may be useful for accurately determining the extent of the inflammatory lesion in the paranasal sinus. Further research is warranted to reduce the time duration required for evaluation using this system, so that in future, it can be employed effectively for monitoring treatment effects in chronic sinusitis and other conditions that involve inflammation of the paranasal sinuses.

\section{References}

[1] Lloyd, G. A., Lund, V. J., and Scadding, G. K. 1991. “CT of the Paranasal Sinuses and Functional Endoscopic Surgery: A Critical Analysis of 100 Symptomatic Patients.” J. Laryngol. 105 (3): 181-5.

[2] Kennedy, D. W. 1992. "Prognostic Factors, Outcomes and Staging in Ethmoid Sinus Surgery.” Laryngoscope 102 (12 Pt2 Supple 57): 1-18.

[3] Young, J., Frenkiel, S., Tewfik, M. A., and Mouadeb, D. A. 2007. "Long-Term Outcome Analysis of Endoscopic Sinus Surgery for Chronic Sinusitis.” Am. J. Rhinol. 21 (6): 743-7.

[4] Lund, V. J., and Mackay, I. S. 1993. "Staging in Rhinosinusitus.” Rhinology 31 (4): 183-4. 


\section{Computed Tomography with Ziostation}

[5] Meltzer, E. O., Hamilos, D. L., Hadley, J. A., Lanza, D. C., Marple, B. F., Nicklas, R. A., Bachert, C., Baraniuk, J., Baroody, F. M., Benninger, M. S., Brook, I., Chowdhury, B. A., Druce, H. M., Durham, S., Ferguson, B., Gwaltney, J. M., Kaliner, M., Kennedy, D. W., Lund, V., Naclerio, R., Pawankar, R., Piccirillo, J. F., Rohane, P., Simon, R., Slavin, R. G., Togias, A., Wald, E. R., and Zinreich S. J. 2004. "Rhinosinusitis: Establishing Definitions for Clinical Research and Patient Care.” J. Allergy Clin. Immunol. 114 (6 Suppl): 155-212.

[6] Kennedy, D. W., Kuhn, F. A., Hamilos, D. L., Zinreich, S. J., Bulter, D., Warsi, G., Pfister P. J., and Tavakkol, A. 2005. "Treatment of Chronic Rhinosinusitis with High-Dose Oral Terbinafine: A Double Blind, PlaceboControlled Study.” Laryngoscope 115 (10): 1793-9.

[7] Okushi, T., Nakayama, T., Morimoto, S., Arai, C., Omura, K., Asaka, D., Matsuwaki, Y., Yoshikawa, M., Moriyama, H., and Otori, N. 2013. "A Modified Lund-Mackay System for Radiological Evaluation of Chronic Rhinosinusitis.” Auris Nasus Larynx 40 (6): 548-53.
[8] Mao, S. S., Li, D., Vembar, M., Gao, Y., Luo, Y., Lam, F., Syed, Y. S., Liu, C., Woo, K., Flores, F., and Budoff, M. J. 2014. "Model-Based Automatic Segmentation Algorithm Accurately Assesses the Whole Cardiac Volumetric Parameters in Patients with Cardiac CT Angiography: A Validation Study for Evaluating the Accuracy of the Workstation Software and Establishing the Reference Values.” Acad. Radiol. 21 (5): 639-47.

[9] Hori, M., Okada, T., Higashiura, K., Sato, Y., Chen, Y. W., Kim, T., Onishi, H., Eguchi, H., Nagano, H., Umeshita, K., Wakasa, K., and Tomiyama, N. 2015. “Quantitative Imaging: Quantification of Liver Shape on CT Using the Statistical Shape Model to Evaluate Hepatic Fibrosis.” Acad. Radiol. 22 (3): 303-9.

[10] Wang, P., Han, X., Yang, G., Zhang, Y., Lan, J., Zhao, H., Qiu, S., Zhen, H., and Gao, Q. 2014. "Computed Tomographic Images Analysis of Frontal Recess Anatomy Based on Three-Dimension Reconstruction.” Journal of Clinical Otorhinolaryngology Head and Neck Surgery 28 (17): 1305-7. 